\title{
Predictors of Prosthetic Valve Endocarditis following Transcatheter Aortic Valve Replacement: A Meta-Analysis
}

\author{
Weiwei Jiang, MD, PhD,${ }^{1^{*}}$ Wenconghui $W u, M S,{ }^{*}$ Ruikang Guo, $M S,{ }^{3}$ Minghui Xie, MS, ${ }^{3}$ Wai Yen Yim, MS, \\ Yin Wang, MD, PhD, ${ }^{3}$ Xingjian $\mathrm{Hu}, \mathrm{MD}, \mathrm{PhD}^{3}$ \\ ${ }^{1}$ Department of Gastroenterology, Union Hospital, Tongji Medical College, Huazhong University of Science and Technology, \\ Wuhan, China; ${ }^{2}$ Department of Gastroenterology, Zhongnan Hospital, Wuhan University, Wuhan, China; ${ }^{3}$ Department of \\ Cardiovascular Surgery, Union Hospital, Tongji Medical College, Huazhong University of Science and Technology, Wuhan, China
}

\section{ABSTRACT}

Background: Transcatheter aortic valve replacement (TAVR) has gained increasing acceptance for patients with aortic disease. A rare but fatal complication prosthetic valve endocarditis (PVE) could greatly influence the clinical outcomes of TAVR. This meta-analysis aims to pin down the predictors of PVE in TAVR patients.

Methods: We performed a systematic search for studies that reported the incidence and risk factors of PVE after TAVR. Data on studies, patients, baseline characteristics, and procedural characteristics were abstracted. Crude risk ratios (RRs) and 95\% confidence intervals for each predictor were calculated by the use of random-effects models. Heterogeneity assumption was assessed by an $\mathrm{I}^{2}$ test.

Results: We obtained data from 8 studies that included 68,805 TAVR patients, of whom 1,256 (1.83\%) were diagnosed with PVE after TAVR. 280 patients died within the 30-days of PVE diagnosis and the pooled in-hospital mortality was $22.3 \%$. The summary estimates indicated an increased risk of PVE after TAVR for males (RR 1.53, $P=.0001)$; for patients with orotracheal intubation (RR 1.65, $P=.01)$, new pacemaker implantation (RR $1.46, P=.003$ ), and residual aortic regurgitation ( $\geq 2$ grade) (RR 1.62, $P=.05$ ); while older age (RR $0.97, P=.0007)$ and implantation of a self-expandable valve (RR $0.74, P=.02$ ) were associated with a lower risk of PVE after TAVR.

Received November 9, 2020; accepted December 1, 2020.

*These authors contributed equally to the work.

This study was financially supported by the National Natural Science Foundation of China (NO.81700317) to Y.W. and (NO. 81400290) to X.H.

Correspondence: Yin Wang, MD, PbD, Department of Cardiovascular Surgery, Union Hospital of Tongji Medical College, Huazhong University of Science and Technology, \#1277 Fiefang Avenue, 430022, Wuban, China; +8613628642979 (e-mail: $2013 x$ b0859@bust.edu.cn).

Xingjian Hu, MD, PhD, Department of Cardiovascular Surgery, Union Hospital of Tongji Medical College, Huazhong University of Science and Technology, \#12777iefang Avenue, 430022, Wuban, China; +8615827346676 (e-mail:peterbu9517@bust.edu.cn).
Conclusion: Clinical characteristics and periprocedure factors including age, male sex, valve type, orotracheal intubation, pacemaker implantation, and residual regurgitation were proven to be associated with the occurrence of PVE-TAVR. Clinicians should pay particular attention to PVE when treating TAVR patients with these predictors.

\section{INTRODUCTION}

Prosthetic valve endocarditis after transcatheter aortic valve replacement (TAVR-PVE) is a relatively rare but fatal complication. The most common pathogens causing TAVR-PVE includ Enterococci, Staphylococcus aureus, and coagulase-negative staphylococci. While some literature suggests the incidence appears to be as low as $0.3 \%$ to $1.2 \%$ per person-year [Misawa 2015; Kolte 2018], the absolute number of cases is likely to rise substantially as TAVR expands into mid and low-risk populations following the publication of the PARTNER 3 and Evolut Low Risk trials [Mack 2019; Braghiroli 2020; Popma 2019]. Treatment of TAVR-PVE largely parallels that of conventional prosthetic valve endocarditis, with prolonged intravenous antibiotic therapy and consideration of surgical intervention forming the cornerstones of management. However, the effect of treatment with surgery or antibiotics is poor, with high mortality rates (in-hospital mortality is $15 \%$ to $30 \%$ ) secondary to heart failure and acute kidney injury [Bax 2019; Tan 2015]. Besides, the early diagnosis of TAVR-PVE is still challenging, given that the presentation is often asymptomatic and the echocardiographic findings are diverse, while robust evidence for specific preventative strategies of this complication is lacking. All these highlight the need for identifying reliable predictors of TAVR-PVE for surgeons or physicians to targeted follow-up and timely intervention. A few researchers focused on this problem and their registry studies based on different populations came to different or even conflicting conclusions. Therefore, we systematically reviewed all studies regarding this topic and employed a meta-analytic strategy to analyze these data with a twofold aim: (i) to determine pooled, final incidence and mortality of PVE in this specific TAVR population, and (ii) to identify perioperative parameters that best discriminated between TAVR patients with and without PVE. 


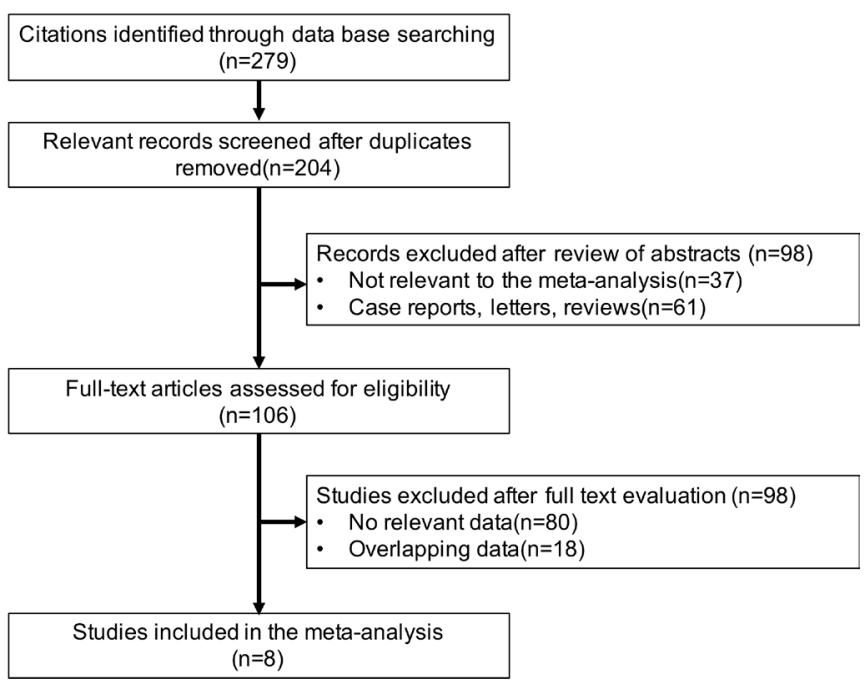

Figure 1. Study selection flow diagram.

\section{MATERIALS AND METHODS}

\section{Search Strategy}

We performed a broad, computerized literature search of certain text and keywords in Medline, EMBASE, and the CENTRAL trials registry of the Cochrane Collaboration through February 2020. The keywords included 'Transcatheter aortic valve implantation', 'Transcatheter aortic valve replacement', 'TAVI', 'TAVR', 'Endocarditis', 'Infective endocarditis', 'Prosthetic valve endocarditis', 'Infective endocarditis after TAVI', 'Incidence and clinical impact of infective endocarditis on TAVI', 'TAVI-associated infective endocarditis', 'Prosthetic valve endocarditis after transcatheter valve replacement', 'Causative organisms of post-TAVI infective endocarditis', 'Clinical outcomes of infective endocarditis after TAVI', 'In-hospital mortality', 'Mortality at follow-up', 'Transcatheter heart failure', and 'Outcomes of TAVI.'

\section{Inclusion and Exclusion Criteria}

The inclusion criteria were as follows: (a) studies illustrating the incidence and risk factors of PVE after TAVR, reporting odds ratios (ORs), risk ratios (RRs) or hazard ratios (HRs), and (b) cohort studies including patients with PVE and patients without PVE. Studies were excluded for the following reasons: (a) the studies were abstracts, editorials, letters, reviews, comments, or case reports, (b) the sample size was less than 20, (c) the studies utilized duplicate databases, or (d) the studies did not include human subjects. If ORs were reported by univariate and multivariate analysis simultaneously, only multivariate ORs were included. Two investigators independently conducted the literature searches, the study eligibility assessment, and the data extraction in duplicate. Any discrepancies were resolved by consensus and arbitration by a third investigator.

\section{Data Extraction and Quality Assessment}

The following study- and patient-related information was extracted from the main paper and any accompanying

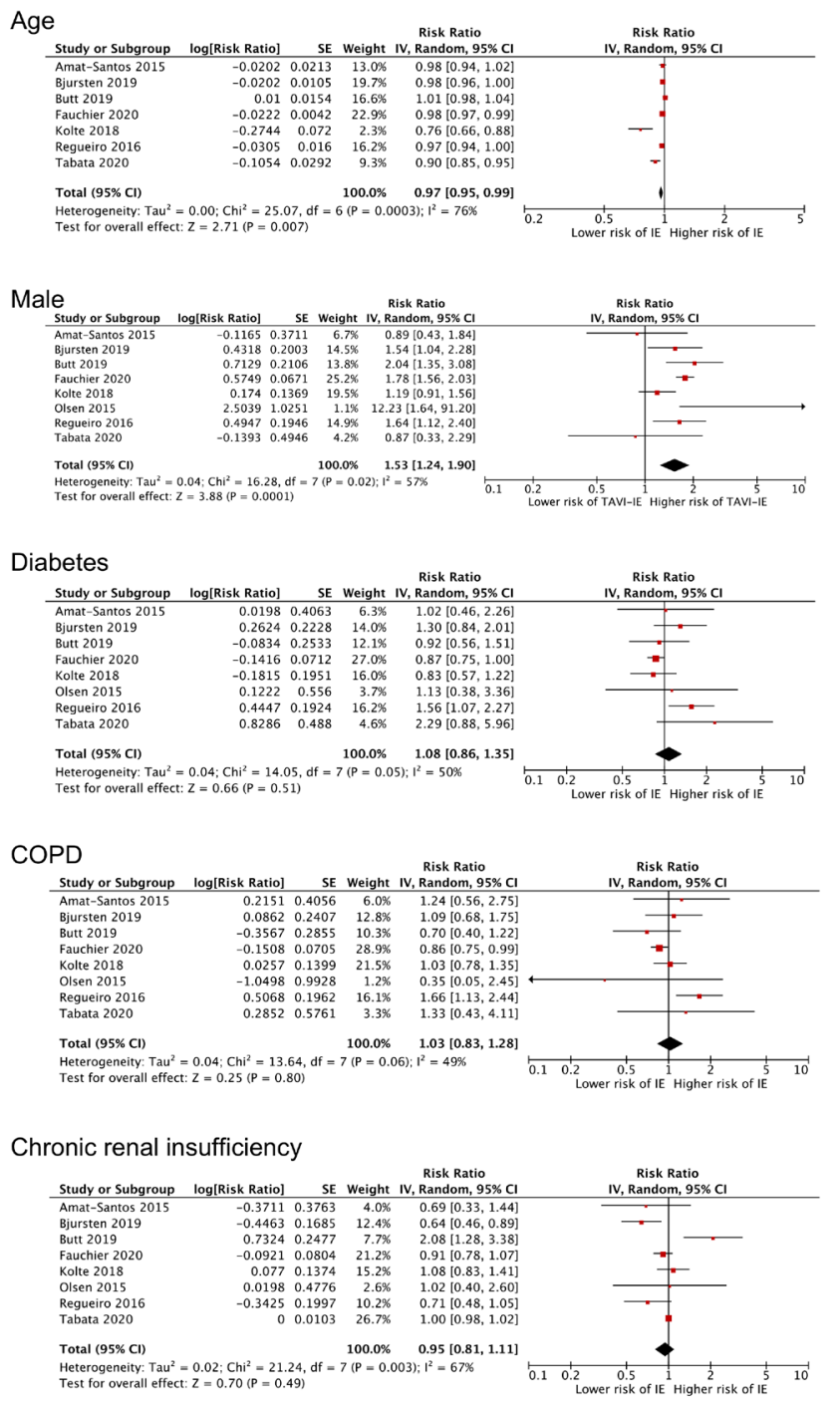

Figure 2. Forest plot of summary crude RRs of each assessed preoperative predictor for patients with prosthetic valve endocarditis after transcatheter aortic valve replacement: age; male sex; diabetes; COPD; chronic renal insufficiency by evaluated IV random-effects model. Heterogeneity estimates $\left(\mathrm{I}^{2}\right)$ are given for those predictors for which datasets from 2 or more studies were available. $\mathrm{Cl}$ indicates confidence interval; IV, inverse variance.

supplemental material: publication year; study design; study period; follow-up duration; number of participants; number of PVE after TAVR; age; male sex; diabetes; chronic obstructive pulmonary disease; chronic renal insufficiency; LogEuroSCORE (\%); mean gradient; valve orifice area; aortic regurgitation ( $\geq$ moderate); left ventricular ejection fraction; valve embolization; self-expandable valve; transfemoral approach; orotracheal intubation; aortic regurgitation ( $\geq$ moderate); left ventricular ejection fraction; valve embolization; pacemaker implantation; vascular injury; acute kidney injury; residual aortic regurgitation ( $\geq$ grade 2 ); blood transfusion; low implantation, and bleeding complication. The quality of the 
Aortic regurgitation $\geq$ moderate

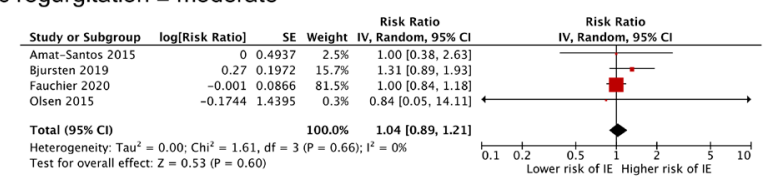

Self-Expandable valve

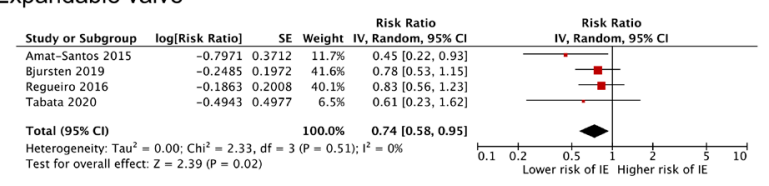

Orotracheal intubation

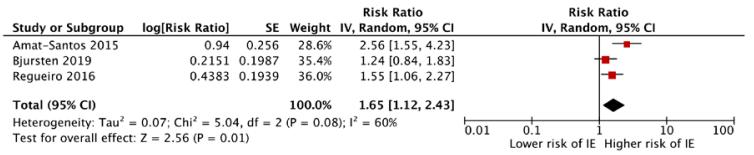

Transfemoral approach

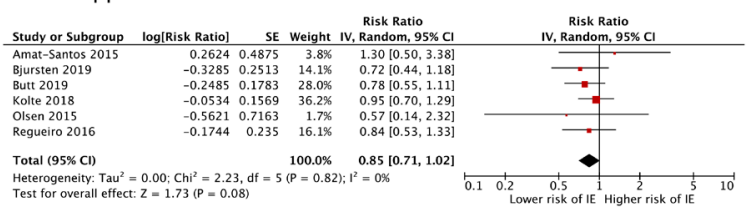

Figure 3. Forest plot of summary crude RRs of each assessed procedural related predictor for patients with prosthetic valve endocarditis after transcatheter aortic valve replacement: aortic regurgitation $\geq$ moderate, self-expandable valve, orotracheal intubation, transfemoral approach by evaluated IV random-effects model. Heterogeneity estimates $\left(I^{2}\right)$ are given for those predictors for which datasets from 2 or more studies were available. $\mathrm{Cl}$ indicates confidence interval; IV, inverse variance.

included studies was assessed by the Newcastle-Ottawa scale.

\section{Assessed Predictors of PVE after TAVR}

According to a recently published review, we focused on the following previously proposed predictors: age; male sex; diabetes; COPD; chronic renal insufficiency; self-expandable valve; transfemoral approach; orotracheal intubation; aortic regurgitation ( $\geq$ moderate); pacemaker implantation; vascular injury; residual aortic regurgitation ( $\geq$ grade 2); and bleeding complication.

\section{Data Analysis}

We pooled RRs using RevMan Software Version 5.3 (The Cochrane Collaboration, London, United Kingdom). The inverse variance method was used to pool multivariate ORs and HRs. Only two or more ORs and HRs were pooled. Heterogeneity across the studies was quantified with the $\mathrm{I}^{2}$ index: an $\mathrm{I}^{2}$ of $0-25 \%$ renders insignificant heterogeneity; 26-50\% low heterogeneity; $51-75 \%$ moderate heterogeneity; and $>75 \%$ high heterogeneity; and a random-effects model was used to obtain the combined effect estimates [Higgins 2002]. Two-sided $P$-values less than .05 were considered statistically significant. Publication bias analysis was performed when pooled studies were more than three. If significant
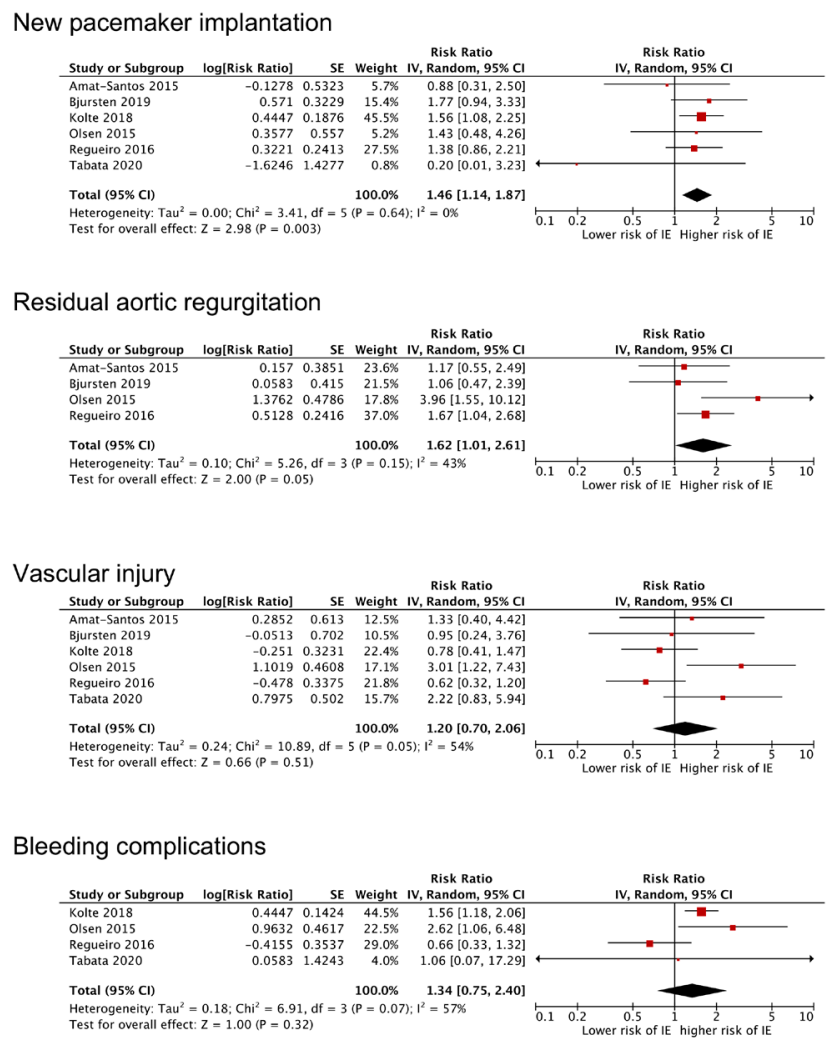

Figure 4. Forest plot of summary crude RRs of each assessed postoperative predictor for patients with prosthetic valve endocarditis after transcatheter aortic valve replacement: residual aortic regurgitation $\geq$ grade 2, vascular injury, bleeding complications by evaluated IV random-effects model. Heterogeneity estimates $\left(\mathrm{I}^{2}\right)$ are given for those predictors for which datasets from 2 or more studies were available. $\mathrm{Cl}$ indicates confidence interval; IV, inverse variance.

publication bias was noted, Duval and Tweedie's trim and fill method was used to acquire adjusted values. The present systematic review and meta-analysis was conducted according to the Preferred Reporting Items for Systematic Reviews and Meta-Analyses (PRISMA) group [Liberati 2009].

\section{RESULTS}

The study selection process is presented in Figure 1. A total of 279 citations were retrieved after searching PubMed, EMBASE, and CENTRAL database. There were 106 fulltext articles assessed for eligibility after screening titles and abstracts, and we identified 35 studies that reported the incidence of PVE after TAVR. Finally, 8 articles [Kolte 2018; Amat-Santos 2015; Olsen 2015; Bjursten 2019; Fauchier 2020; Tabata 2020; Butt 2019; Regueiro 2016] were ultimately included in the present systematic review and metaanalysis. The main characteristics and the overall quality of the included studies are shown in Table 1. Overall, 68,805 patients were evaluated in 8 studies, and 1,256 (1.83\%) were diagnosed with PVE after TAVR. The incidence of PVE after 
Table 1. Characteristics of Included Studies

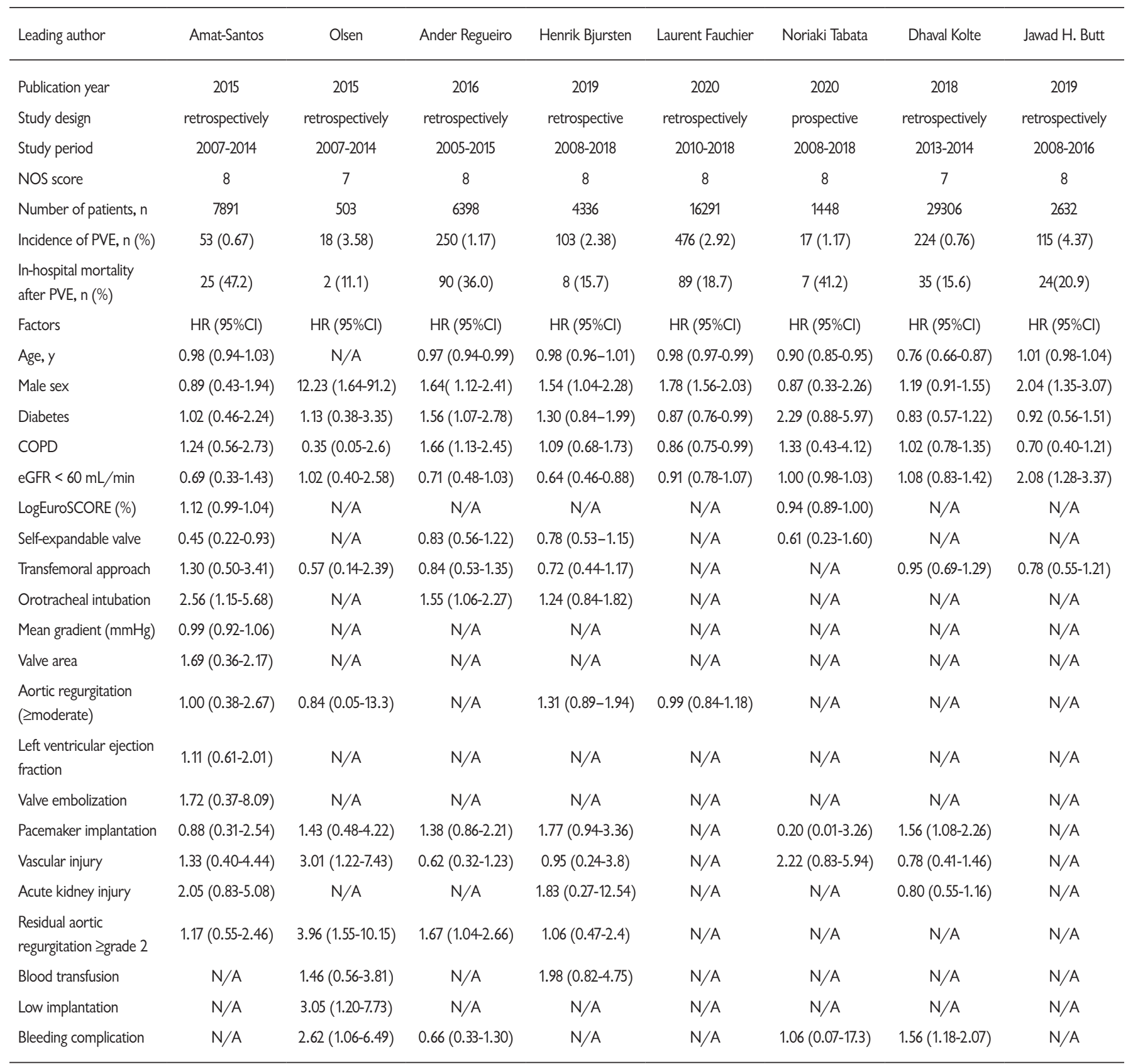

$\mathrm{Cl}$ indicates confidence interval; COPD, chronic obstructive pulmonary disease; eGFR, estimate glomerular filtration rate; HR, hazard ratio; NOS, NewcastleOttawa scale; PVE, prosthetic valve endocarditis.

the intervention ranged from $0.7 \%$ to $4.3 \%$ in individual studies. The pooled in-hospital mortality was $22.3 \%(280 / 1256)$, ranged from $11.1 \%$ to $47.2 \%$ in individual studies.

\section{Baseline Characteristics-Related Factors}

Older age [Kolte 2018; Amat-Santos 2015; Bjursten 2019; Fauchier 2020; Tabata 2020; Butt 2019; Regueiro 2016] was associated with a lower risk of PVE after TVAR (RR 0.97,
95\% CI: 0.95 to $0.99, P=.007$ ), and men sex [Kolte 2018; Amat-Santos 2015; Olsen 2015; Bjursten 2019; Fauchier 2020; Tabata 2020; Butt 2019; Regueiro 2016] was identified as a predictor of PVE after TVAR (RR 1.53, 95\% CI: 1.24 to $1.90, P=.0001$ ), while diabetes [Kolte 2018; Amat-Santos 2015; Olsen 2015; Bjursten 2019; Fauchier 2020; Tabata 2020; Butt 2019; Regueiro 2016] (RR $1.08,95 \%$ CI: 0.86 to $1.35, P=.51$ ), COPD [Kolte 2018; 
Table 2. Test of Heterogeneity and Publication Bias for Each Outcome

\begin{tabular}{|c|c|c|c|c|c|c|c|c|}
\hline Male sex & 8 & 68805 & IV, Random & $1.53(1.24,1.90)$ & .0001 & $57 \%$ & moderate & none \\
\hline Diabetes & 8 & 68805 & IV, Random & $1.08(0.86,1.35)$ & .51 & $50 \%$ & low & none \\
\hline Aortic regurgitation ( $\geq$ moderate) & 4 & 29027 & IV, Random & $1.04(0.89,1.21)$ & .6 & $0 \%$ & insignificant & none \\
\hline Self-expandable valve & 4 & 20073 & IV, Random & $0.74(0.58,0.95)$ & .02 & $0 \%$ & insignificant & none \\
\hline Transfemoral approach & 6 & 51072 & IV, Random & $0.85(0.71,1.02)$ & .08 & $0 \%$ & insignificant & none \\
\hline Orotracheal intubation & 3 & 18625 & IV, Random & $1.65(1.12,2.43)$ & .01 & $60 \%$ & moderate & none \\
\hline Bleeding complication & 4 & 37661 & IV, Random & $1.34(0.75,2.40)$ & .32 & $57 \%$ & moderate & none \\
\hline
\end{tabular}

$\mathrm{Cl}$ indicates confidence interval; COPD, chronic obstructive pulmonary disease; HR, hazard ratio; IV, inverse variance.

Amat-Santos 2015; Olsen 2015; Bjursten 2019; Fauchier 2020; Tabata 2020; Butt 2019; Regueiro 2016] (RR 1.03, $95 \%$ CI: 0.83 to $1.28, P=.80$ ), chronic renal insufficiency [Kolte 2018; Amat-Santos 2015; Olsen 2015; Bjursten 2019; Fauchier 2020; Tabata 2020; Butt 2019; Regueiro 2016] (RR $0.95,95 \%$ CI: 0.81 to $1.11, P=.49$ ) and aortic regurgitation $\geq$ moderate [Amat-Santos 2015; Olsen 2015; Bjursten 2019; Fauchier 2020] (RR 1.04, 95\% CI: 0.89 to $1.21, P=.60)$ were not correlated with PVE after TVAR (Figure 2).

\section{Procedure-Related Factors}

A self-expandable valve [Amat-Santos 2015; Bjursten 2019; Tabata 2020; Regueiro 2016] was associated with a lower risk of PVE after TVAR (RR 0.74, 95\% CI: 0.58 to $0.95, P=.02$ ) and orotracheal intubation [Amat-Santos 2015; Bjursten 2019; Regueiro 2016] was identified as a predictor of PVE after TVAR (RR $1.65,95 \%$ CI: 1.12 to $2.43, P=.01$ ), while transfemoral approach [Kolte 2018; Amat-Santos 2015; Olsen 2015; Bjursten 2019; Butt 2019; Regueiro 2016] was not correlated with PVE after TVAR approach (RR 0.85, 95\% CI: 0.71 to $1.02, P=.08$ ) (Figure 3 ).

\section{Post-TAVI Characteristics}

Patients with a new pacemaker implantation [Kolte 2018; Amat-Santos 2015; Olsen 2015; Bjursten 2019; Tabata 2020; Regueiro 2016] (RR 1.46, 95\% CI: 1.14 to $1.87, P=$ $.003)$ and more than grade 2 residual aortic regurgitation [Amat-Santos 2015; Olsen 2015; Bjursten 2019; Regueiro 2016] (RR 1.62, 95\% CI: 1.01 to $2.61, P=.05$ ) after TAVR appeared to be more susceptible to PVE. Vascular injury [Kolte 2018; Amat-Santos 2015; Olsen 2015; Bjursten 2019;
Tabata 2020; Regueiro 2016] (RR 1.2, $95 \%$ CI: 0.70 to 2.06, $P=.51)$ and bleeding complications [Kolte 2018; Olsen 2015; Tabata 2020; Regueiro 2016] (RR 1.34, 95\% CI: 0.75 to $2.4, P=.32$ ) were not associated with PVE after TVAR (Figure 4). The results of each factor were summarized in Table 2. No significant publication biases were detected for each individual analysis.

\section{DISCUSSION}

Transcatheter aortic valve replacement (TAVR) has emerged as an accepted alternative to surgical aortic valve replacement (SAVR). Its effect has been carefully evaluated in several RCT studies. The most recent PARTNER 3 study showed that TAVR was superior to SAVR in preventing death, stroke, and repeat hospitalization at one-year follow-up [Mack 2019]. Similarly, the EVOLUT Low Risk trial showed no difference in two-year outcomes (mortality, stroke) between SAVR and TAVR [Popma 2019]. Based on these new RCT trials, the indication for TAVR continues to shift toward patients at a younger age or lower risk [Thyregod 2015; Waksman 2018]. These patients will have a longer postoperative survival period in which may develop subsequent infective endocarditis on the prosthesis and require recurrent interventions. Although the incidence of PVE was proved to be rare in previous studies and the present metaanalysis (pooled incidence: $1.62 \%$ ), the occurrence of TAVRPVE is likely to become more frequent as implant numbers rise rapidly. Besides, we must keep in mind that the current diagnostic criteria for definite infective endocarditis such as Duke or modified Duke criteria are known to be less sensitive 
in PVE [Kuttamperoor 2019]. That is why some guidelines recommended 18-fluorodeoxyglucose PET or singlephoton emission CT in the diagnosis of PVE to increase sensitivity. In addition, the incidence of para-valvular abscesses and fistulas emanating from the infected prosthetic valve is higher than PVE after SAVR [Ben-Shoshan 2016]. Moreover, TAVR-PVE can also lead to other complications include heart block, heart failure, renal failure, and septic shock and worsened mortality. On the other hand, the therapeutic methods for TAVR-PVE are quite limited. Consequently, the outcomes of TAVR-PVE are very unpromising. If prompt intervention is not undertaken, this severe complication in patients can ultimately prove fatal (pooled inhospital mortality: 22.3\%) [Bax 2019; Tan 2015; Butt 2019]. Identification of predictors can facilitate targeted screening during follow-up and timely intervention, which has great value in clinical practice.

However, several studies based on single/multi-center data or different registries provide various and contradictory predisposing indicators for TAVR-PVE. The discrepancy may pose questionable advice to the clinical practice. To our best knowledge, this is the first meta-analysis aimed at identifying independent predictors of PVE in TAVR patients. 13 possible predisposing indicators were extracted from 8 trials, and according to currently available evidence, younger age, male sex, valve design, orotracheal intubation, pacemaker implantation, and residual moderate or severe regurgitation appeared correlated with the risk of subsequent endocarditis occurrence after TAVR. Although some highly speculative predisposing indicators, such as diabetes mellitus, COPD, access approach, or chronic renal dysfunction, have been identified as risk factors in some previous studies, they were proven to have no significant effects on the occurrence of TAVR-PVE in the present meta-analysis.

As shown in the present meta-analysis, the pool rate of PVE after TAVR was $1.83 \%$; conversely the average incidence of PVE after SAVR was only 0.57\% [Glaser 2017]. We speculate the remaining atheroma after TAVR plays an important role in the occurrence of TAVR-PVE. Additionally, the other reason may be the factor that biological prostheses are more reluctant to PVE compared with mechanical valves [Anantha-Narayanan 2020]. Finally, the present metaanalysis shows that the pooled postoperative 30-day mortality was $22 \%$; it seems that SAVR-PVE has a lower postoperative 30-day mortality (13\%) than TAVR-PVE [Manne 2012].

Some nonprocedural risk factors such as age and sex were related to the development of PVE after TAVR. Younger patients are more likely to develop PVE after TAVR, while male patients had 1.5 times higher risk for the development of prosthesis infection. Some researchers suggested that younger patients that have undergone TAVI commonly have a higher comorbidity burden, which might correlate with susceptibility to infections of the prosthesis [Tabata 2020; Regev 2017]. One possible explanation for the differences in sex is the female protective theory based on the endothelial protection effect of estrogen. Nevertheless, further fundamental studies are required to reveal the underlying mechanisms. Age and sex are two risk factors one cannot modify, but there are other modifiable factors associated with the development of TAVR-PVE. Self-expandable valve (SEV) was less prone to have endocarditis compared with balloon-expandable valve $(\mathrm{BEV})$. The differences between these two systems in terms of the design, loading processing of prosthetic valve, and delivery system and procedure may help explain this phenomenon [Regueiro 2019; Abdel-Wahab 2016]. The mechanism could be related to the different delivery techniques as BEV implantation could cause greater tissue damage secondary to balloon dilatation during prosthetic valve deployment. It is worth mentioning that in the study of Amat-Santos et al [Amat-Santos 2015], the authors identified SEV as an independent risk factor for PVE after TAVR by univariate analysis (HR, 4.56; 95\% Cl, 2.34-10.3; $P=.025$ ). However, according to the supplemental material, there were 3067 patients enrolled, and 29 patients were diagnosed with PVE. Among these 29 patients with IE, 16 patients $(55.2 \%)$ received a SEV, while in 3028 patients without PVE, 2229 patients $(73.6 \%)$ received a SEV. The calculated HR for PVE with SEV in the univariate analysis should be 0.45 with $95 \%$ CI $0.218-0.933$ $(P=.032)$, which leads to just the opposite of the conclusion of the paper. So we only adopted the primary data from this paper for meta-analysis. Orotracheal intubation is another predictor for TAVR-PVE. Orotracheal intubation indicated general anesthesia, mechanical ventilation, and more complicated and invasive procedures that may increase bacteremia and subsequent prosthesis contamination. TAVR procedure often requires a temporary pacemaker insertion. In addition, due to the pressure of an expandable stent on left ventricle outflow tract, TAVR patients had a higher incidence of heart block compared to SAVR [Young Lee 2015] and required a permanent pacemaker be implanted. Pacemakers have been shown to be a nidus for infection and might lead to the development of PVE. The crude delivery of the device is another potential avenue for infection. Residual moderate or severe aortic regurgitation is related to prosthesis-annulus size mismatch, technical difficult procedures with prosthesis malpositioning, and is more common when treating heavily and asymmetrically calcified aortic valves. Residual aortic regurgitation may predispose to developing endocarditis because of the high-velocity regurgitate jet damages or otherwise increase in the vulnerability of endothelial surfaces [Athappan 2013]. The pockets between the prosthesis and native valve allow for thrombus formation, which then can serve as a nidus for infection [Kuttamperoor 2019].

\section{Study Limitations}

Several limitations to the current meta-analysis need to be acknowledged. The main limitation is the design of included studies because both retrospective and prospective observational studies are exposed to several biases. Although we performed a thorough assessment of their methodological quality, the risk of bias being inherent to the study design cannot be ruled out completely. The use of different generation transcatheter prostheses from various manufacturers in these studies may also limit the validity of the findings in the present meta-analysis, since there are certain, albeit minor, differences in different prostheses. Finally, the data analyzed in this 
study are all observational with no randomized trials, leading to an indication bias. However, in the shortage of randomized data, the findings of our meta-analysis can further advise the practice of TAVR clinicians and influence future studies.

\section{Conclusion}

Despite being infrequent, PVE after TAVR is a deadly complication associated with high rates of mortality. Several clinical characteristics, such as age, male sex, valve design, orotracheal intubation, pacemaker implantation, and residual regurgitation, show adequate specificity for the occurrence of TAVR-PVE. This finding raises a flag of warning that surgeons and physicians should perform TAVR with particular attention of PVE in the specific patient cohort. As TAVR indications continue to expand to lower risk and younger patients, consensus guidelines should clarify the appropriate diagnosis and management of PVE after TAVR.

\section{REFERENCES}

Abdel-Wahab M, Neumann FJ, Mehilli J, et al. 2015. 1-year outcomes after transcatheter aortic valve replacement with balloon-expandable versus self-expandable valves: Results from the choice randomized clinical trial. J Am Coll Cardiol 66:791-800.

Amat-Santos IJ, Messika-Zeitoun D, Eltchaninoff H, et al. 2015. Infective endocarditis after transcatheter aortic valve implantation: Results from a large multicenter registry. Circulation 131:1566-74.

Anantha-Narayanan M, Reddy YNV, Sundaram V, et al. 2020. Endocarditis risk with bioprosthetic and mechanical valves: Systematic review and meta-analysis. Heart 106:1413-19.

Athappan G, Patvardhan E, Tuzcu EM, et al. 2013. Incidence, predictors, and outcomes of aortic regurgitation after transcatheter aortic valve replacement: Meta-analysis and systematic review of literature. J Am Coll Cardiol 61:1585-95.

Bax JJ, Delgado V. 2019. Prosthetic valve endocarditis after surgical and transcatheter aortic valve replacement: Infrequent, but poor outcome. Euro Intervention 15:e484-5.

Ben-Shoshan J, Amit S, Finkelstein A. 2016. Transcatheter aortic valve implantation infective endocarditis: Current data and implications on prophylaxis and management. Curr Pharm Des 22:1959-64.

Bjursten H, Rasmussen M, Nozohoor S, et al. 2019. Infective endocarditis after transcatheter aortic valve implantation: A nationwide study. Eur Heart J 40:3263-9.

Braghiroli J, Kapoor K, Thielhelm TP, Ferreira T, Cohen MG. 2020. Transcatheter aortic valve replacement in low risk patients: A review of partner 3 and EVOLUT low risk trials. Cardiovasc Diagn Ther 10:59-71.

Butt JH, Ihlemann N, De Backer O, et al. 2019. Long-term risk of infective endocarditis after transcatheter aortic valve replacement. J Am Coll Cardiol 73:1646-55.

Fauchier L, Bisson A, Herbert J, et al. 2020. Incidence and outcomes of infective endocarditis after transcatheter aortic valve implantation versus surgical aortic valve replacement. Clin Microbiol Infect 26:1368-74.

Glaser N, Jackson V, Holzmann MJ, Franco-Cereceda A, Sartipy U. 2017. Prosthetic valve endocarditis after surgical aortic valve replacement.
Circulation 136:329-31.

Higgins JP, Thompson SG. 2002. Quantifying heterogeneity in a metaanalysis. Stat Med 21:1539-58.

Kolte D, Goldsweig A, Kennedy KF, et al. 2018. Comparison of incidence, predictors, and outcomes of early infective endocarditis after transcatheter aortic valve implantation versus surgical aortic valve replacement in the united states. Am J Cardiol 122:2112-9.

Kuttamperoor F, Yandrapalli S, Siddhamsetti S, Frishman WH, Tang GHL. 2019. Infectious endocarditis after transcatheter aortic valve replacement: Epidemiology and outcomes. Cardiol Rev 27:236-41.

Liberati A, Altman DG, Tetzlaff J, et al. 2009. The prisma statement for reporting systematic reviews and meta-analyses of studies that evaluate healthcare interventions: Explanation and elaboration. BMJ 339:b2700.

Mack MJ, Leon MB, Thourani VH, et al. 2019. Transcatheter aorticvalve replacement with a balloon-expandable valve in low-risk patients. N Engl J Med 380:1695-705.

Manne MB, Shrestha NK, Lytle BW, et al. 2012. Outcomes after surgical treatment of native and prosthetic valve infective endocarditis. Ann Thorac Surg 93:489-93.

Misawa Y. 2015. Valve-related complications after mechanical heart valve implantation. Surg Today 45:1205-9.

Olsen NT, De Backer O, Thyregod HG, et al. 2015. Prosthetic valve endocarditis after transcatheter aortic valve implantation. Circ Cardiovasc Interv 8:e001939.

Popma JJ, Deeb GM, Yakubov SJ, et al. 2019. Transcatheter aortic-valve replacement with a self-expanding valve in low-risk patients. $\mathrm{N}$ Engl J Med 380:1706-15.

Regev E, Finkelstein A, Assali A, et al. 2017. Comparison of outcome of transcatheter aortic valve implantation for severe aortic stenosis in 3 age groups $(</=70 ; 71$ to 80 , and $>/=81$ years $)$. Am J Cardiol 120:1607-11.

Regueiro A, Linke A, Latib A, et al. 2016. Association between transcatheter aortic valve replacement and subsequent infective endocarditis and in-hospital death. JAMA 316:1083-92.

Regueiro A, Linke A, Latib A, et al. 2019. Infective endocarditis following transcatheter aortic valve replacement: Comparison of balloonversus self-expandable valves. Circ Cardiovasc Interv 12:e007938.

Tabata N, Al-Kassou B, Sugiura A, et al. 2020. Predictive factors and long-term prognosis of transcatheter aortic valve implantation-associated endocarditis. Clin Res Cardiol 109:1165-76.

Tan HL, Chai LY, Yeo TC, Chia BL, Tambyah PA, Poh KK. 2015. Predictors of in-hospital adverse events in patients with prosthetic valve infective endocarditis. Heart Lung Circ 24:705-9.

Thyregod HG, Steinbruchel DA, Ihlemann N, et al. 2015. Transcatheter versus surgical aortic valve replacement in patients with severe aortic valve stenosis: 1-year results from the all-comers notion randomized clinical trial. J Am Coll Cardiol 65:2184-94.

Waksman R, Rogers T, Torguson R, et al. 2018. Transcatheter aortic valve replacement in low-risk patients with symptomatic severe aortic stenosis. J Am Coll Cardiol 72:2095-105.

Young Lee M, Chilakamarri Yeshwant S, Chava S, Lustgarten LD. 2015. Mechanisms of heart block after transcatheter aortic valve replacement - cardiac anatomy, clinical predictors and mechanical factors that contribute to permanent pacemaker implantation. Arrhythm Electrophysiol Rev 4:81-5. 